\title{
Dissociation Mechanism of 6-APA reacted with a varying number of $\mathrm{H}^{+}$ Cui Shuling ${ }^{a}$, Ning Yuxi ${ }^{b}$ \\ Hebei University of Science and Technology, Shijiazhuang 050018, China \\ aemail: cuishulingling@126.com, bemail: nyx100@126.com
}

Keywords: 6-APA; Density Function Theory; beta lactam; ring-opening

\begin{abstract}
Properties of the products derived from the reaction of 6-aminopenicillanic acid (6-APA) with varying number of $\mathrm{H}^{+}$have been researched by B3LYP method of the Density Function Theory (DFT) on the basis of 6-31G* sets. It is found that the products are all unstable, and easily open their beta lactam rings. The ring-opening pathway is closely related to the $\mathrm{H}^{+}$number with which 6-APA reacted. Three ring-opening pathways of 6-APA were found by calculation, and the energy barrier of the three ring-opening pathways are respectively $37.54 \mathrm{~kJ} / \mathrm{mol}, 32.61 \mathrm{~kJ} / \mathrm{mol}$ and $2.63 \mathrm{~kJ} / \mathrm{mol}$. Meanwhile we got the transition state structures and their unique negative imaginary frequency values. The correctness of the result was proved by intrinsic reaction coordinate (IRC) method.
\end{abstract}

\section{Introduction}

There is only one mechanism reported about the ring-opening reaction of 6-aminopenicillanic acid (6-APA) in domestic and abroad literature at present, which is the breaking of carbon-nitrogen bond taking place on the four-member-ring of beta lactam. As for the transition state in the ring-opening process, only the formation process of transition state has been speculated, while the accurate structure of the transition state and the reaction barrier have not been reported so far ${ }^{[1-4]}$.

The structure of 6-APA consists of two parts: one is the four-member-ring of beta lactam, and the other is five- member-ring of hydrogenated thiazole. The tension of 4-member-ring is relatively larger, so the beta lactam is easier to take place ring-opening, hydrolyzing or rearrange when encountering acid, alkali, nucleophiles, electrophiles, some metal ions and oxidizing substances or reductive agents. As a result, a series of by products are generated which may lose the antibacterial activity of 6-APA, increases its drug anaphylaxis, and even threat life of human body if the by-products content exceeds the standard. This paper will research the products derived from the reaction of 6-aminopenicillanic acid (6-APA) with varying number of $\mathrm{H}^{+}$by quantum chemistry calculation so as to explore the ring-opening mechanism and provide theoretical support for synthesizing high purity of penicillin products.

\section{Methods of the research}

B3LYP method of the Density Function Theory (DFT) on the basis of 6-31G* sets was used in this paper. The transition state structures were found through analyzing vibration frequency and gradient calculation. Meanwhile the correctness of transition state structures were verified through intrinsic reaction coordinate (IRC) calculation. All of the works were finished by using the Program Gaussian 03.

\section{Description of spatial structure and character of 6-apa}

The structure and character of 6-apa was researched first. The spatial structure of 6-apa was optimized. It was found that 6-apa had a free carboxyl group and the charge density of each atom was calculated. Considering carboxyl easily rotating in the space, the figure of rotating potential was made by calculating the rotation barriers of carboxyl in 6-APA.

It can be known from the character of spatial structure of 6-APA (see Fig.1 and Fig.2) that the $\pi$ bond of carbonyl cannot conjugate with the lone pair electrons of nitrogen atom in the beta lactam, 
so the carbon atom in the carbonyl is easily attacked by nucleophilic reagents while the oxygen in the carbonyl and the nitrogen atoms in beta lactam are easily attacked by eletrophilic reagent. It can be seen from Fig. 2 that there are two stable points with energy close to each other in the carboxyl group, as means that carboxyl can rotate during the reaction process, and the rotating process can provide valuable scope of stable state during searching transition state. The various transition state and intermediate structures are shown in Fig 3.

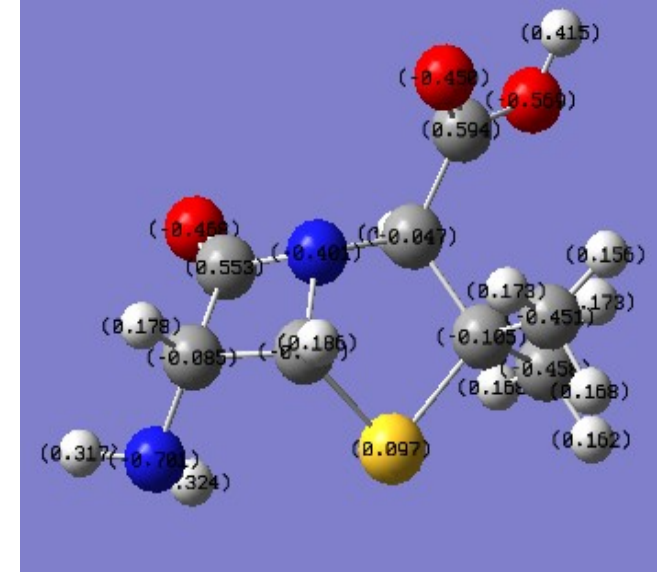

Fig.1 Space structure and charge distribution of 6-APA

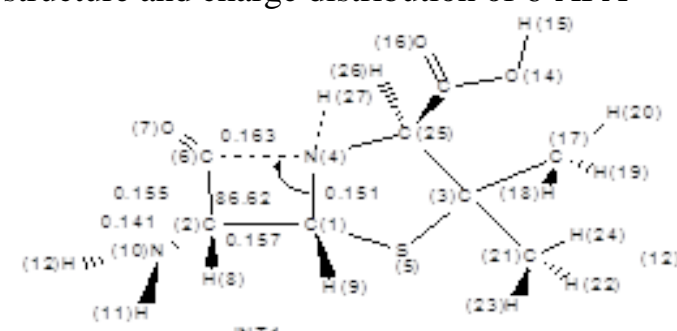

$\mathrm{NT} 1$

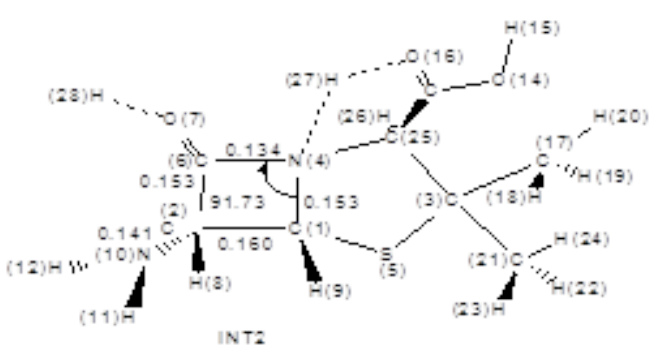

INT2

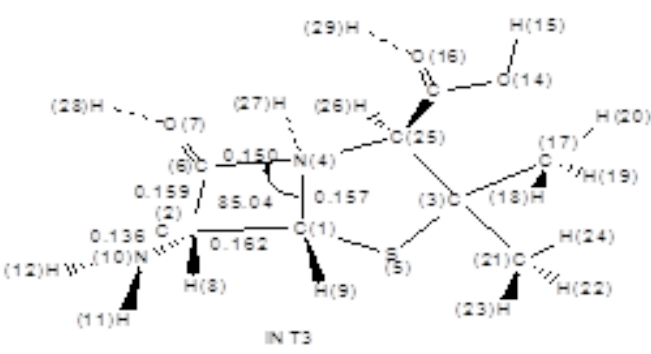

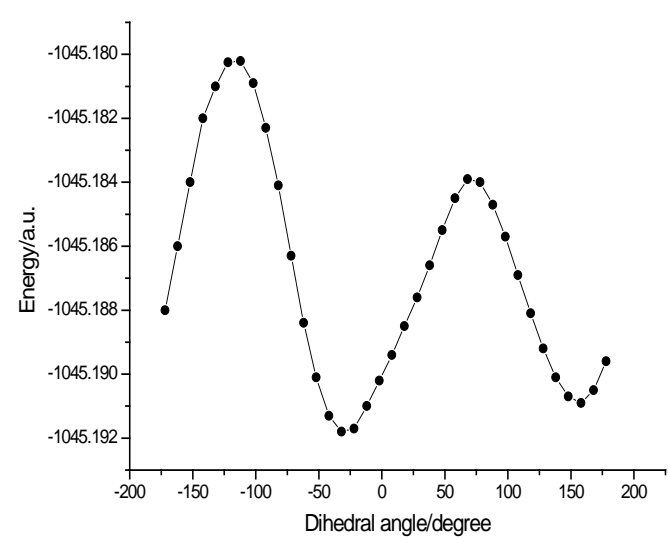

Fig. 2 The rotation barriers of carboxyl in 6-APA
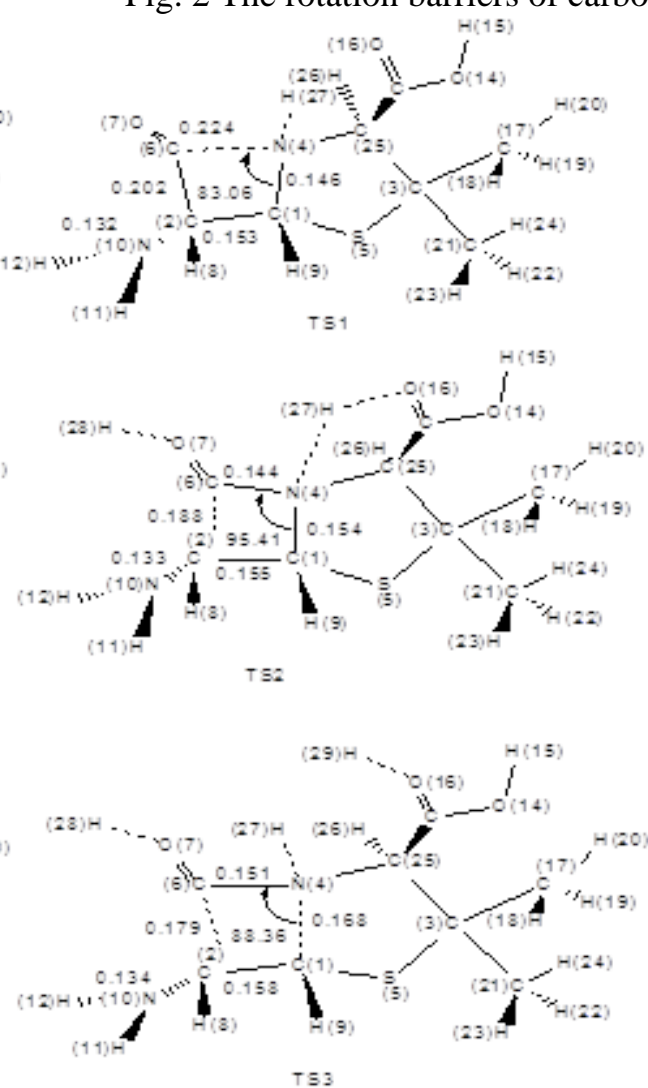

Fig. 3 Optimized geometric parameters of transition states and intermediates (bond length/nm; bond angle/degree)

\section{The spatial structures of transition state}

The transition state structures are found through analyzing vibration frequency and gradient calculation. At the same time the structures of transition state are checked through intrinsic reaction coordinate (IRC) calculation. To observe intuitively the spatial structures of transition state, the stereostructures and energy variations of IRC during the reaction process are respectively diagramed (see Fig.4, Fig.5, and Fig.6). 

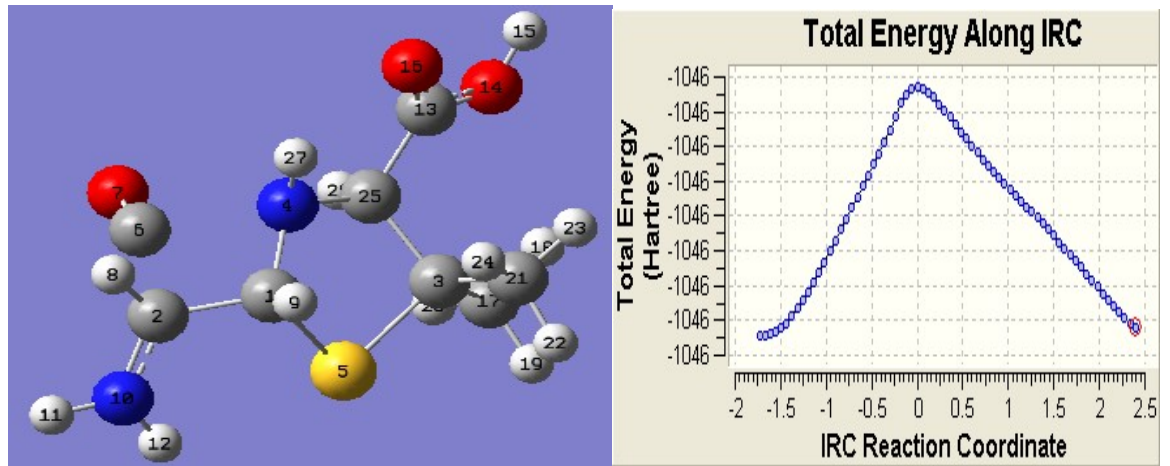

Fig.4 Transition state and IRC of 6-APA reacted with one $\mathrm{H}^{+}$
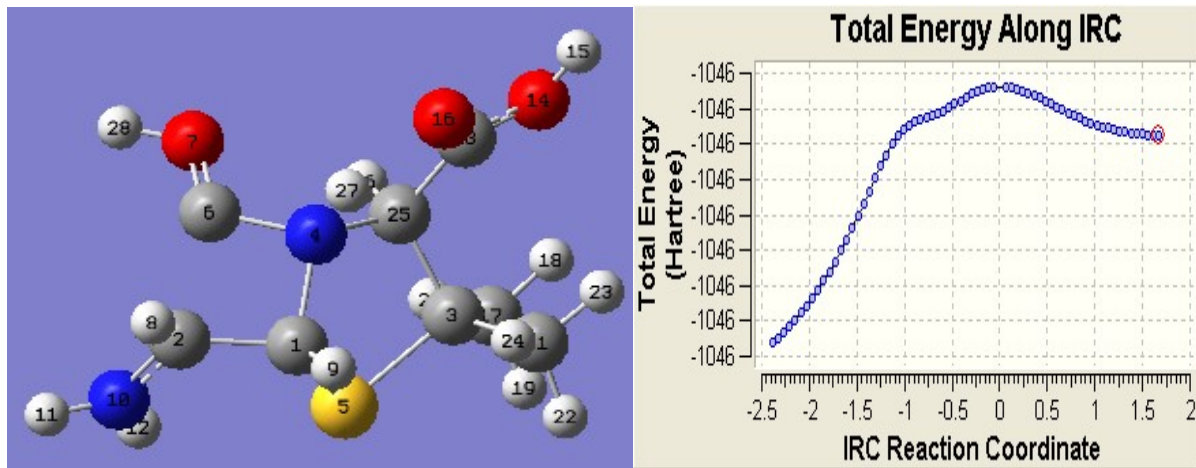

Fig.5 Transition state and IRC of 6-APA reacted with two $\mathrm{H}^{+}$
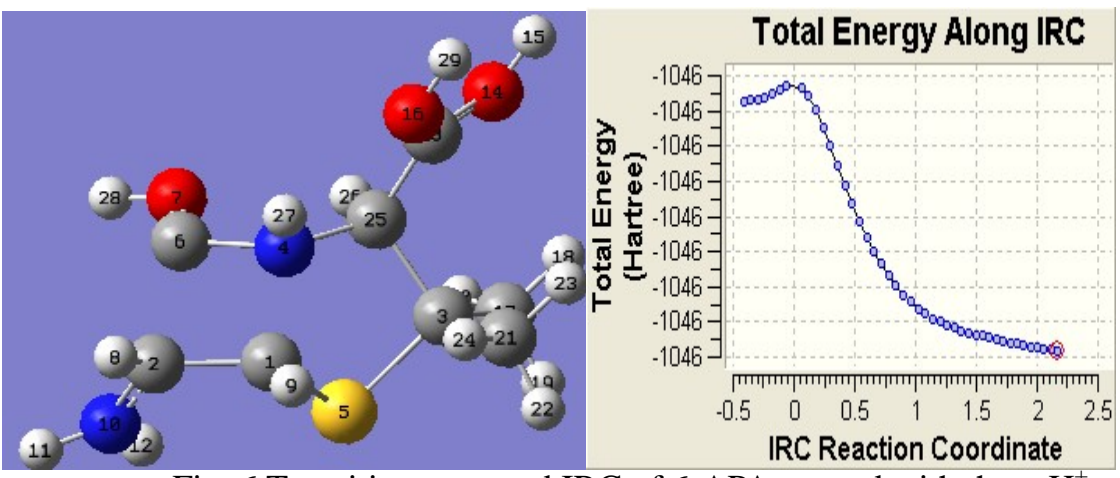

Fig. 6 Transition state and IRC of 6-APA reacted with three $\mathrm{H}^{+}$

\section{Energy data analysis}

The corresponding Energy data of various transition states, intermediates and products are respectively shown in Table 1.

Table 1 Total and relative energy of transition states, intermediates and products (INT1 as the reference point)

\begin{tabular}{ccc}
\hline & Total energy/a.u. & ${\text { Relative energy } / \mathrm{kJ}^{\cdot} \mathrm{mol}^{-1}}^{-1}$ \\
INT1 & -1045.7789 & 0 \\
TS1 & -1045.7646 & 37.54 \\
P1 & -1045.7889 & -26.26 \\
INT2 & -1045.9283 & -394.93 \\
TS2 & -1045.9169 & -362.32 \\
P2 & -1045.9238 & -380.43 \\
INT3 & -1045.9675 & -495.17 \\
TS3 & -1045.9665 & -492.54 \\
P3 & -1045.9817 & -532.45
\end{tabular}

By the analytical data from table 1, it can be found that the energy barrier of the three ring-opening pathways are respectively $37.54 \mathrm{~kJ} / \mathrm{mol}, 32.61 \mathrm{~kJ} / \mathrm{mol}$ and $2.63 \mathrm{~kJ} / \mathrm{mol}$. 


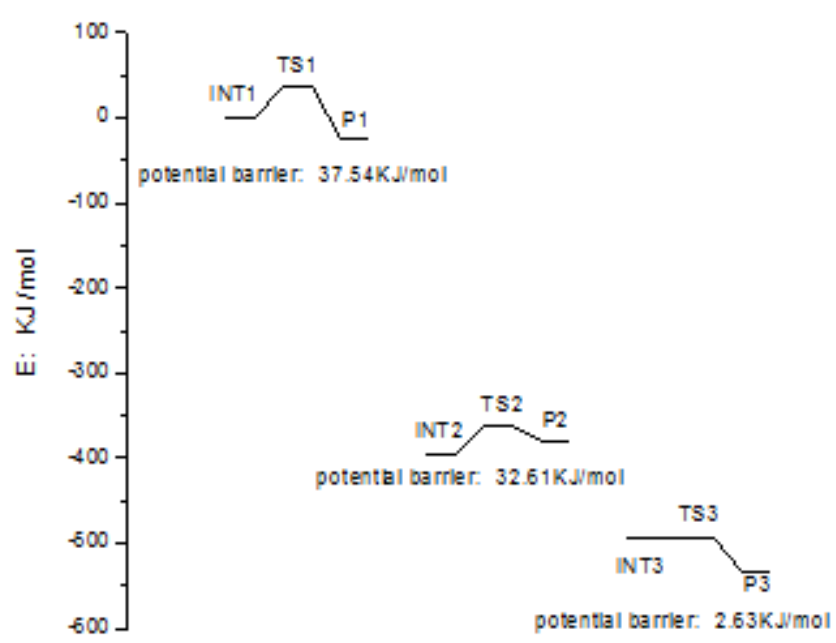

Fig.7 Reaction potential barriers

By analyzing above figures of transition state structures, IRC and reaction potential barriers, it is obvious that the ring-opening pathway is closely related to the $\mathrm{H}^{+}$number reacted with 6-APA. When 6-APA reacts with one $\mathrm{H}^{+}$, the ring-opening process is slow and its mechanism is the breaking of carbon-nitrogen bond. The reaction barrier energy is high and the product is stable which cannot further dissociate. When 6-APA reacts with two $\mathrm{H}^{+}$, the ring-opening process is quick with mechanism of carbon-carbon bond breaking. The reaction barrier energy is lower than the first one and the product is not stable. When 6-APA reacts with three $\mathrm{H}+$, the four-member-ring as well as the five-member-ring broken simultaneously and quickly. At this moment, the properties of the products is very unstable, showing very low energy reaction barrier. Moreover the energy of products is much lower than that of reactant. This phenomena demonstrate that 6-APA is very active in strong acid condition and will decompose into many stable products, so the reaction process is difficult to control.

\section{Conclusion}

The reaction mechanism of 6-aminopenicillanic acid (6-APA) with varying number of $\mathrm{H}^{+}$was researched by quantum chemistry calculation. There are three ring-opening pathways in the reaction process. which is closely related to the $\mathrm{H}^{+}$number with which 6-APA reacted, and the energy barrier of the three ring-opening pathways are respectively $37.54 \mathrm{~kJ} / \mathrm{mol}, 32.61 \mathrm{~kJ} / \mathrm{mol}$ and $2.63 \mathrm{~kJ} / \mathrm{mol}$. The correctness of the results were proved by intrinsic reaction coordinate (IRC) method.

\section{References}

[1] A. L1jnas, M. I. Page. Intramolecular General Acid Catalysis in the Aminolysis of $\beta$-Lactam Antibiotics [J].Org.Biomo1.Chem.2004 2 651-654.

[2] J.Pitarch,J.L.Pascual Ahuir,E.Siila. Modelingß-Lactam Interactions in Aqueous Solution through Combined Quantum Mechanics - Molecular Mechanics Method [J]. Journal of Computational Chemistry.1999 20(13) 1401-1411.

[3] N.Diaz, D.Sudrez, T.L.Sordo, I.Tuh6n, E.Silla. Water-assisted Alkaline Hydrolysis of Monobactams:A Theoretical Study [J].Chem-Eur.J.2002 8 859-867.

[4] G.L.Boroskya, F.Munoz.Chemical Reactivity of Oxo-and Aza $\sim \beta$ - Lactam Rings[J]. Helve.Chimica Acta.,2001 84 526-534. 